\title{
SPACE-BASEd ERBIUM-DOPED FibER AMPLIFIER TrANSMITTERS FOR Coherent, Ranging, 3D-IMaging, Altimetry, Topology, AND Carbon Dioxide Lidar and EARTH AND Planetary Optical Laser CoMmunications Mark Storm*, Doruk Engin, Brian Mathason, Rich Utano, and Shantanu Gupta
}

Fibertek, Inc., 13605 Dulles Technology Drive, Herndon, VA 20171, USA, *mstorm@fibertek.com

\begin{abstract}
This paper describes Fibertek, Inc.'s progress in developing space-qualified Erbium-doped fiber amplifier (EDFA) transmitters for laser communications and ranging/topology, and $\mathrm{CO}_{2}$ integrated path differential absorption (IPDA) lidar. High peak power $(1 \mathrm{~kW})$ and $6 \mathrm{~W}$ of average power supporting multiple communications formats has been demonstrated with $17 \%$ efficiency in a compact $3 \mathrm{~kg}$ package. The unit has been tested to Technology Readiness Level (TRL) 6 standards. A $20 \mathrm{~W}$ EDFA suitable for $\mathrm{CO}_{2}$ lidar has been demonstrated with $\sim 14 \%$ efficiency (electrical to optical [e-o]) and its performance optimized for $1571 \mathrm{~nm}$ operation.
\end{abstract}

\section{INTRODUCTION}

Fibertek has been developing eye-safe 1.5-um lidars and associated laser technology for numerous applications including coherent wind lidar [1,2]; 3D imaging and ranging for obstacle avoidance, docking, brownout, and rendezvous lidars [3,4]; $\mathrm{CO}_{2}$ lidar [5]; and deep space, low/geosynchronous earth orbit (LEO/GEO) laser communications [6,7]. Many of our applications require specialized multi-stage designs to provide unique performance such as high 1-3 mJ laser pulse energies [8], kilohertz linewidth, absolute wavelength control, differential absorption lidar (DIAL), $\mathrm{CO}_{2}$ lidar, or modulation formats for chirped pulse ranging lidar or pseudo-random noise lidar [9]. Multi-stage amplifiers are often needed to achieve 30-60 dB of net gain.

Commercial off-the-shelf (COTS) EDFA amplifiers are typically 1-10 W single stage units and used for ground-based laboratory testing and pressurized aircraft cabin lidar measurements. Systems requiring specialized multi-stage performance require customized assemblies of amplifiers and other optical components and can get large, unwieldy, and unsuitable for UAV or spaceflight platforms where small size and specialized performance are needed. Electronics and packaging can make COTS components and assemblies challenging.

This paper describes our progress in engineering EDFA transmitters into a spaceflight/UAV quality package that operates in challenging environments, has traceability, and is vibration and thermal vacuum tested toward TRL-6 qualification.

\section{METHODOLOGY}

Fibertek develops fiber lasers based on the performance specifications, environment of operation, and expected reliability and lifetime of the mission. After the application performance is specified Fibertek conducts an optical design and breadboard validate phase to optimize and validate the design. A team of engineers then conducts an optical, thermal, and structural design using models and finite element analysis. The design is iterated to optimize the package performance and then built and environmentally tested. Analysis products including thermal, structural finite element model (FEM), and random vibration spectrum and modal analysis are shown in Figure 1.

\section{RESULTS}

\subsection{Deep Space, GEO, and LEO Laser Communications Transmitter}

Fibertek is developing a space-qualifiable, highly efficient high-power ( $>5 \mathrm{~W}$ ) fiber-based $1550 \mathrm{~nm}$ laser optical module (LOM) supporting NASA JPL. The JPL developed deep space comm system is being made available on the NASA Discovery 2014 mission instrument announcement of opportunity. The system is designed to operate from deep space, planets, and Mars and the transmitter is also suitable for high-reliability LEO/GEO communications (Figure 2). The transmitter achieves $6 \mathrm{~W}$ average and up to $1 \mathrm{~kW}$ peak power depending on the communications pulse format $[6,7]$. 


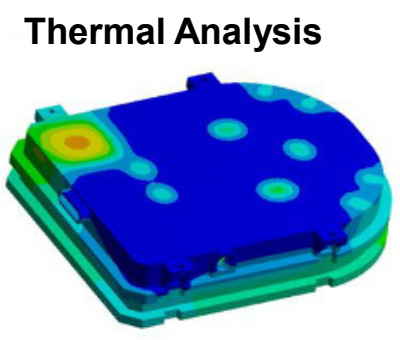

\section{Structural FEMA Analysis}

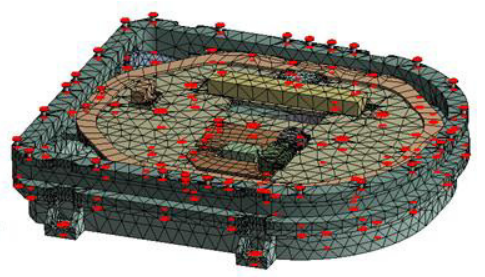

\section{Random Vibration}

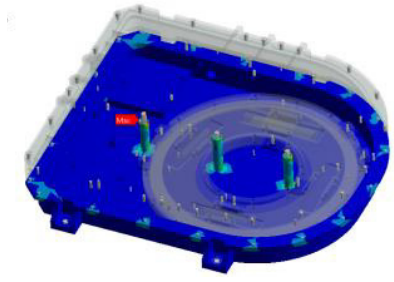

Figure 1: Thermal, Structural, and Radom Vibration Analyses Meet or Exceed Margins of Safety for Launch and Survival and Operation of Mission Temperature Ranges.

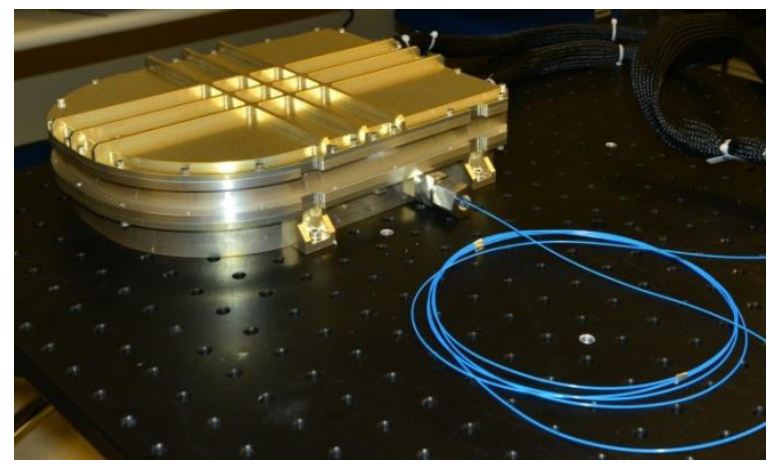

Figure 2: Overall Laser Optical Module

The transmitter is also suitable for ranging/topology because the peak powers are high. The transmitter has $<6 \mathrm{GHz}$ linewidth. Stimulated Brillouin scattering (SBS) is managed by precise linewidth control and use of large mode array (LMA) gain fiber in the power stage while maintaining the required diffraction limited and highly polarized (polarization extinction ratio [PER] $>20 \mathrm{~dB}$ ) output. Size and weight of the transmitter are $8^{\prime \prime} \times 10^{\prime \prime} \times 2.4^{\prime \prime}$ and $3 \mathrm{~kg}$, respectively. The unit includes the distributed feedback (DFB) laser diodes, EO modulators, and amplifier.

With improvements in the modulation scheme and component specification, the achieved e-o efficiency is more than $17 \%$. Highly efficient operation is sustained for a wide range of pulseposition modulation (16 to 128-ary PPM) formats with pulsewidths varying from $8 \mathrm{nsec}$ to $0.5 \mathrm{nsec}$ and operation temperature $10-50^{\circ} \mathrm{C}$. Pressure stress, random vibration, and thermal analyses of the designed LOM predict compliance with NASA General Environmental Verification Standard (GEVS) levels for vibration and thermal cycling in a vacuum environment. The transmitter has undergone and passed TRL- 6 thermal vacuum and vibration testing to validate the design.

\subsection{High-Efficiency $\mathrm{CO}_{2}$ Amplifier Performance at $1571 \mathrm{~nm}$ for IPDA Lidar}

Fibertek is also developing a continuous wave (CW) EDFA at $1571 \mathrm{~nm}$ for $\mathrm{CO}_{2}$ IPDA (Figure 3). IPDA is a two-way integration of the opticaldepth (OD) from platform to surface (e.g., ground or cloud top), and is measured using lock-in detection of an intensity-modulated $\mathrm{CW}$ fiber laser transmitter employing multiple wavelengths within a $\mathrm{CO}_{2}$ absorption linewidth of $\sim 1 \mathrm{GHz}$ $(\sim 10 \mathrm{pm})$ [10-12].

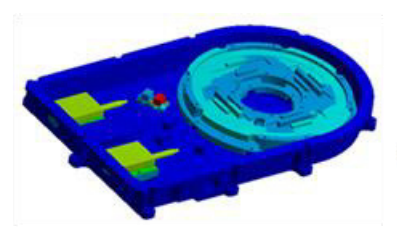

Thermal Analysis Blue is $20 \mathrm{C}$, Red is $39 \mathrm{C}$

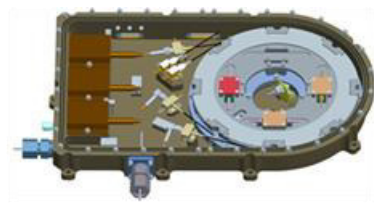

Size: 14 " x 8.5" x 2" Mass: $3.7 \mathrm{~kg}$

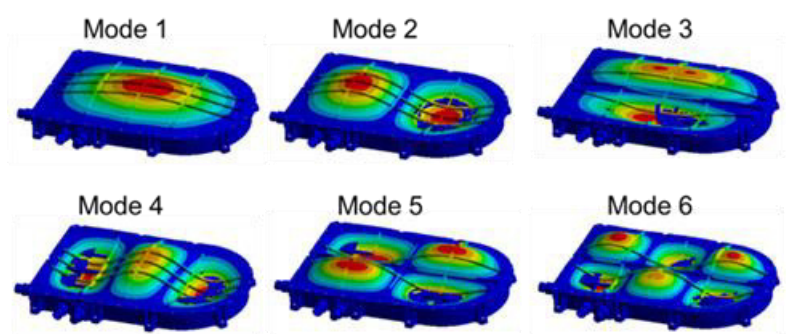

Vibration modes for GEVs 14.1 grms. All main structural modes $>2000 \mathrm{~Hz}$

Figure 3: Solid Model of the Design and Preliminary Thermal and Structural Analysis for 20 W Average Power EDFA Amplifier 
This two-stage amplifier can also support the coherent lidar DIAL method [13]. This spaceflight TRL-6 prototype transmitter is custom designed to meet NASA $\mathrm{CO}_{2}$ lidar requirements that is far advanced compared to current demonstrated space or terrestrial lasers.

The optical transmitter diagram for the $1571 \mathrm{~nm}$ EDFA is a fiber laser master oscillator power amplifier (MOPA) with an ultra-low-noise master oscillator and two-stage fiber amplifiers. The MOPA seed consists of two DFB laser diodes operated $\mathrm{CW}$ representing the lidar on and off wavelengths. The DFB operates with a linewidth of $<1 \mathrm{MHz}$ and can be tuned on and off the $\mathrm{CO}_{2}$ absorption line at $1571.11 \mathrm{~nm}$.

The stage 1 EDFA is built with an Er/Ytterbium (Yb) co-doped single-mode fiber that is widely used in telecom systems. It has core and cladding diameters of $7 \mu \mathrm{m}$ and $125 \mu \mathrm{m}$, and is counterpumped with a broad area laser diode at $976 \mathrm{~nm}$. The stage 1 amplifier maximum $\mathrm{CW}$ power is $\sim 1$ W. For a more detailed description see ref 5.

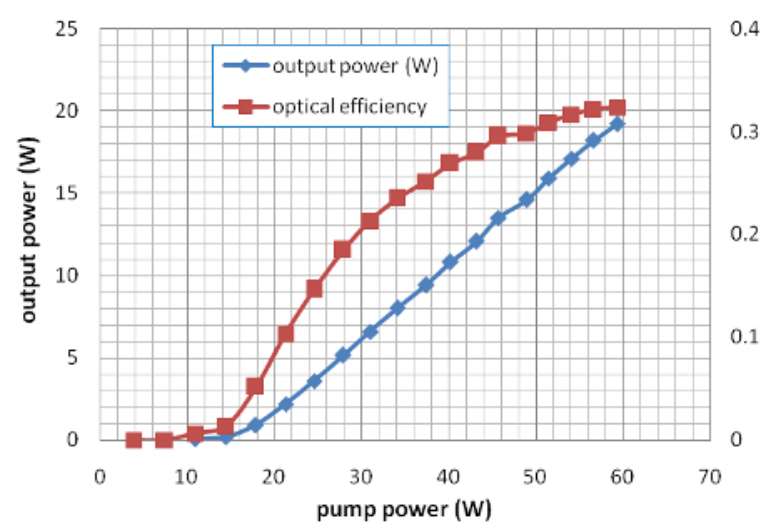

The second stage amplifier employs a commercial LMA Er/Yb co-doped fiber with cladding-pump scheme. Simple coiling of the gain fiber enables single-mode operation. We achieved maximum output power of $21 \mathrm{~W}$ after band-pass filter, and the optical-to-optical efficiency is about $36 \%$ as shown in Figure 4 (left). On the right is the full spectrum after the ASE filter. The signal-to-noise ratio (SNR) is more than $30 \mathrm{~dB}$. The backward spectrum (not shown) does not show any significant SBS nonlinearity.

\subsection{Space-Qualified Yb MOPA for LISA and ICESat-2}

Fibertek has also developed and made significant progress in qualifying a $\mathrm{Yb}$ MOPA transmitter. Figure 5 shows a multi-stage $\mathrm{Yb}$ MOPA fiber amplifier capable of up to $20 \mathrm{~W}$ of power. The $\mathrm{Yb}$ MOPA was successfully vibration tested, thermally cycled, and radiation tested to $2 \mathrm{x}$ mission dosage. The unit was developed as part of the ICESat-2 design effort and designed for 400 $\mathrm{uJ} /$ pulse at $10 \mathrm{kHz}$ repetition rate.

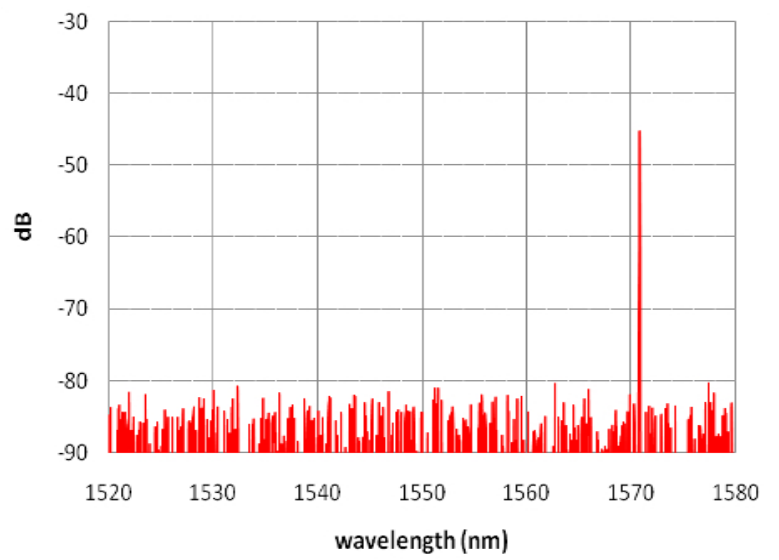

Figure 4: Final Stage Performance Measured After ASE Filter (left) and Forward Spectrum After ASE Filter with $0.01 \mathrm{~nm}$ Resolution (right)
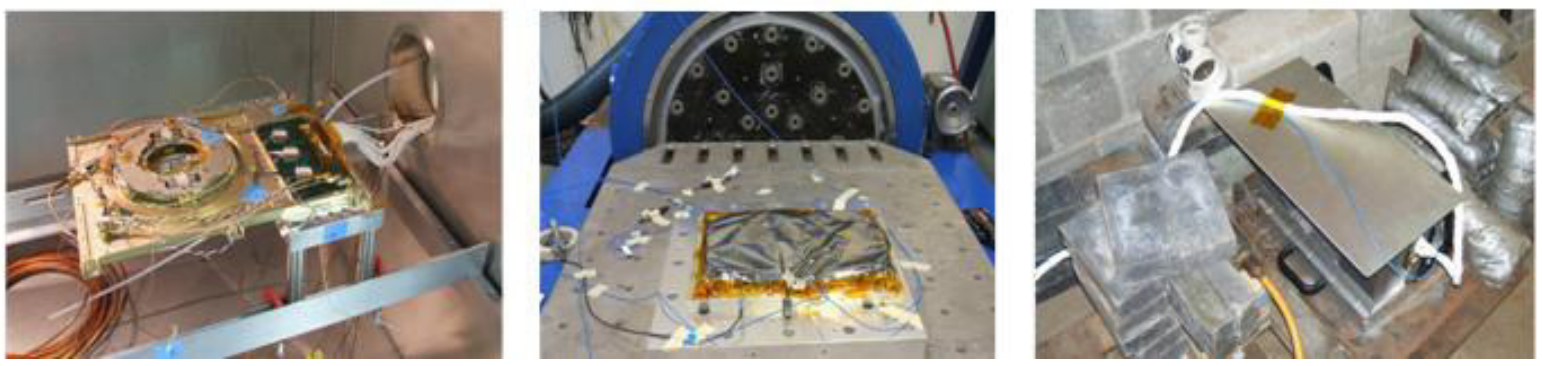

Figure 5: Space Qualification of a Yb MOPA Fiber Amplifier - in Thermal Chamber (left), on a Vibration Table (center), and Being Radiation Tested (right) 
The $\mathrm{Yb}$ amplifier spaceflight transmitter was developed in support of the joint NASA-European Space Agency (ESA) Laser Interferometer Space Antenna (LISA) project to develop and operate a space-based gravitational wave detector.

\section{CONCLUSIONS}

In this paper, we demonstrate that the spaceflight EDFA MOPA and Yb MOPA fiber laser transmitter packages have been developed and environmentally tested to TRL-6 standards. The transmitters developed are high efficiency and capable of supporting high peak powers for laser communications, can be operated at $1571 \mathrm{~nm}$ with single-frequency transform limited performance, and are being prepared for spaceflight use. The laser communications unit supports the NASA Discovery 2014 AO. The $1571 \mathrm{~nm}$ EDFA supports the $\mathrm{CO}_{2}$ IPDA lidar and the NASA ASCENDS 2007 Earth Science Decadal survey mission. The fiber laser generates $20 \mathrm{~W}$ CW power at $1571 \mathrm{~nm}$ with narrow linewidth (1-10 $\mathrm{MHz}$ ). The optical-to-optical efficiency is $36 \%$.

\section{ACKNOWLEDGMENT}

The authors would like to acknowledge the support of NASA. We would also like to acknowledge the work of Fibertek engineers Mehmet Akbulut and Wei $\mathrm{Lu}$ for their early contributions to this work.

\section{REFERENCES}

[1] Akbulut M., et al., "Pulsed coherent fiber lidar transceiver for aircraft in-flight turbulence and wake-vortex hazard detection," Proc. SPIE 8037, Laser Radar Technology and Applications XVI, 80370R (7 June 2011); doi: 10.1117/12.883990.

[2] Akbulut M., "Fiber Laser Coherent Lidar for Wake-Vortex Hazard Detection," 16th Coherent Laser Radar Conference, 2011.

[3] Gary Stevenson, Horacio R. Verdun, Peter H. Stern, Walter Koechner, "Testing the Helicopter Obstacle Avoidance System," Proc. SPIE 2472, Applied Laser Radar Technology II; doi: 10.1117/12.212025.

[4] Thomas A. DuBois, Charles A. DiPietro, Gary Stevenson, "System concept for a rotorcraft vision system to improve cargo-handling operations," SPIE Proceedings Enhanced and Synthetic Vision, Volume 2736.

[5] Lu W., "High-power, narrow linewidth 1.5- $\mu \mathrm{m}$ fiber amplifier lidar transmitter for atmospheric CO2 detection," Proc. SPIE 8961, Fiber Lasers
XI: Technology, Systems, and Applications, 89610C (March 7, 2014); doi:10.1117/12.204120.

[6] Engin D., et al., "Highly reliable and efficient $1.5 \mu \mathrm{m}$ fiber-MOPA-based, high-power laser transmitter for space communication," Proc. SPIE 9081, Laser Technology for Defense and Security $X$, 90810G (9 June 2014); doi: 10.1117/12.2050496.

[7] Engin D., et al., "Highly efficient and athermal $1550 \mathrm{~nm}$ fiber-MOPA-based high-power down link laser transmitter for deep space communication," Proc. SPIE 8610, Free-Space Laser Communication and Atmospheric Propagation XXV, 86100G (19 March 2013); doi: 10.1117/12.2005926.

[8] Torruellas, Y. Chen, B. McIntosh, J. Farroni, K. Tankala, et al., "High peak power ytterbiumdoped fiber amplifiers," Proc. SPIE 6102, Fiber Lasers III: Technology, Systems, and Applications, 61020N; doi:10.1117/12.646571; http://dx.doi.org/10.1117/12.646571.

[9] Xiaoli-Xiaoli Sun and James B. Abshire, "Modified PN code laser modulation technique for laser measurements," Proc. SPIE 7199, FreeSpace Laser Communication Technologies XXI, 71990P (February 24, 2009); doi:10.1117/12.817254; http://dx.doi.org/10.1117/12.817254

[10] E. Browell, K. Davis, J. Abshire, J. Dobler, G. Ehert, P. Flamant, D. Sakaizawa, U. Singh, G. Spiers, "Current Status of Active Remote Sensing of $\mathrm{CO} 2$ and Applications to NACP Investigations," 3rd North American Carbon Program Meeting, New Orleans, Louisiana (1-4 February 2011).

[11] James B. Abshire, et al., "A lidar approach to measure $\mathrm{CO} 2$ concentrations from space for the ASCENDS Mission," Proc. SPIE 7832; doi:10.1117/12.868567; http://dx.doi.org/10.1117/12.868567

[12] D. Sakaizawa, S. Kawakami, T. Tanaka, M. Nakajima, "Improvement of the 1.57 um laser absorption sensor with chirp modulation to evaluate spatial averaging carbon dioxide density," Proc. SPIE, Vol. 8182 (2011).

[13] G. D. Spiers, R. T. Menzies, J. Jacob, L. E. Christensen, M. W. Phillips, Y. Choi, E. V. Browell, "Atmospheric $\mathrm{CO} 2$ measurements with a 2-um airborne laser absorption spectrometer employing coherent detection," Applied Optics 50 (14), pp. 2098-2111 (May 2011). 